\title{
URETHRAL EXTENSIBILITY APPLIED TO RECONSTRUCTIVE SURGERY
}

\author{
E. ALEXSANDRO DA SILVA AND FRANCISCO J. B. SAMPAIO*
}

From the Urogenital Research Unit, State University of Rio de Janeiro, Rio de Janeiro, Brazil

\section{ABSTRACT}

Purpose: The human male urethra has great capacity to extend under traction. This extensibility is the principle of some surgical techniques used to overcome urethral defects and for penile lengthening. However, to our knowledge safe limits of urethral extensibility in reconstructive surgery have not been yet established by a morphological study. To this end we measured fresh human cadaveric urethras with and without traction.

Materials and Methods: We analyzed the macroscopically normal, fresh cadaveric urethra from 25 men 6 months to 73 years old at death (mean age 30.6). Penile length and length of the total, penile, bulbar and membranous urethra were measured. Length under maximal constant traction and the maximal stretched length without penile curvature at artificial erection were determined. Extensibility is expressed as the percent of the variation in initial length at rest and maximal constant traction length. Maximal stretched length without penile curvature at artificial erection is expressed as a percent of maximal constant traction length.

Results: Total urethral extensibility was higher than penile extensibility $(\mathrm{p}<0.001)$. Urethral extensibility decreased with aging $(\mathrm{r}=-0.806, \mathrm{p}<0.001)$. Mean extensibility of the whole male urethra was $66.2 \% \pm 7.2 \%$ and differences among urethral segments were not significant $(\mathrm{p}=$ 0.283 ). Mean maximal stretched length without penile curvature at artificial erection was $75.2 \%$ $\pm 3.8 \%$ and it did not change with age.

Conclusions: Knowledge of the safe anatomical limit of urethral extensibility applied to reconstructive surgery may avoid complications and the necessity for more complex techniques. This limit should be approximately $75 \%$ of the maximal constant traction length or a gap-tonormal urethra ratio of 1:4. However, age related variations should be considered.

\section{KEY WORDS: urethra, penis, reconstructive surgical procedures, elasticity}

The human male urethra can be divided into the prostatic, membranous and spongy urethral/segments, which have structural differences. The spongy/urethra, also called the anterior urethra due to its location, consists of an almost central tunnel of urothelial mucosa supported by a network of interconnected spaces lined with endothelium surrounded by a thin fibrous envelope. The lamina propria of the mucosa is loose connective tissue with abundant elastic networks. No independent submucosa layer is distinguishable. The anterior urethra is composed of a large portion of extracellular matrix distributed in a unique structure. Smooth muscle cells and elastic fibers are major components of the extracellular matrix and, in addition to the spongy feature, they provide the human male urethra with a large capacity to extend under traction. ${ }^{1}$ This extensibility is the principle of several surgical techniques used to correct urethral defects and more recently provide a real increase in penile length. ${ }^{2-10}$ While excessive mobilization of the spongy urethra is not desirable, the success rate of these surgical techniques depends on the possibility of forming free tension anastomosis of the stretched urethral segment. Urethral anastomosis under tension can cause complications, such as penile curvature or chordee. Despite long-standing use of the extension properties of the male urethra in reconstructive surgery,,$^{2,3}$ to our knowledge urethral extensibility has not yet been established by a morphological study. To determine the safe limits of male urethra extensibility for reconstruc-

Accepted for publication December 21, 2001.

Supported by grants from the National Council of Scientific and Technological Development, and Foundation for Research Support of Rio de Janeiro.

* Requests for reprints: Urogenital Research Unit-UERJ, Av. 28 de Setembro, 87-fundos-FCM-térreo, Rio de Janeiro, RJ, 20551030, Brazil. tive surgery we measured normal, fresh human cadaveric urethras with and without traction.

\section{MATERIALS AND METHODS}

We studied 28 macroscopically normal, fresh human cadaveric urethras from men who died of causes not related to the urogenital tract. The local committee on human research approved the study. To obtain the urethra all skin around the penis was dissected and an H-shaped fragment of pubic bone was removed. The prostate, urethra and penile shaft were completely removed and frozen at $-20 \mathrm{C}$. After 24 to 48 hours the specimen was dissected at $22 \mathrm{C}$. The individuals were 6 months to 73 years old at death (mean age 30.6 ) and time between death and specimen extraction was 4 to 10 hours (mean 6).

Artificial erection with saline was induced in all specimens ${ }^{11}$ and the erect penis was photographed with a digital camera. The image was transferred to a computer and the angle of penile deviation was determined by the 5-line system ${ }^{12}$ using imaging software. We excluded 3 specimens from study due to penile curvature greater than 15 degrees. During dissection the limits of the urethral segments were marked with a nylon stitch to ensure accurate measurement after traction. To divide the urethra we used certain anatomical limits, including the penile urethra (from the junction of the urethra with the retroglanular sulcus to the median insertion of the bulbospongiosus muscle) bulbar urethra (following the penile urethra to the urethral insertion in the rhabdosphincter), membranous urethra (following the bulbar urethra to the prostatic apex) and total urethra (from urethral meatus to prostatic apex). Penile length was measured from the inferior edge of the pubic arch to the urethral meatus. 
All measurements were obtained with a rigid rule graduated in $\mathrm{mm}$. and the mean of 3 consecutive readings was recorded. Length is expressed in $\mathrm{cm}$. After initial length measurement the urethra was completely mobilized from adjacent structures. Urethral length at rest (unstretched) and under maximal constant manual traction were determined. Extensibility is expressed as the percent variation in maximal constant traction length and urethral length at rest. Also, the maximal stretched length that did not induce penile curvature at saline induced artificial erection was determined. To assess the latter length we used the sum of bulbar and penile maximal constant traction lengths because it represented the total available segment of anterior urethra on which the extension principle can be applied for reconstructive procedures. While still under the same tension used for assessing maximal constant traction, this segment was reattached to the tunica albuginea of the corpora cavernosa and artificial erection was induced with intracavernous saline. Consequently penile curvature was present in all specimens. Urethral tension was progressively decreased until no evidence of penile curvature was detected. This resultant urethral segment, termed the maximal stretched length without penile curvature at artificial erection, was measured. The difference in this segment and maximal constant traction length is expressed as a percent.

To assess differences among groups the $t$ test and analysis of variance were used when appropriate. Extensibility and maximal stretched length without penile curvature at artificial erection changes with age were correlated by linear regression analysis. Data are expressed as the mean \pm 1 standard deviation with $\mathrm{p}<0.05$ considered statistically significant.

\section{RESULTS}

Mean unstretched penile length was $11.0 \pm 2.8 \mathrm{~cm}$. After applying maximal constant traction it was $16.5 \pm 4.1 \mathrm{~cm}$. Penile extensibility was $51.1 \% \pm 7.0 \%$.

Mean whole urethral length before and after maximal constant traction was $18.6 \pm 5.0$ and $30.7 \pm 8.1 \mathrm{~cm}$., respectively. Mean urethral extensibility was $66.2 \% \pm 7.2 \%$. Differences among the distinct urethral segments were not significant (table 1). Urethral extensibility was significantly greater than penile extensibility $(\mathrm{p}<0.001)$ and each value decreased with aging (fig. 1). The mean critical value of maximal stretched length without penile curvature at artificial erection was $75.2 \% \pm 3.8 \%$, which slightly decreased with aging. Due to its poor correlation coefficient it was considered a constant factor (fig. 2).

To apply our results in clinical practice we expressed the problem by the equation, Un $=$ Smax - Def, where Un represents the safe length of normal urethra in $\mathrm{cm}$. to be mobilized and extended, Smax is the maximum stretched length that did not induce penile curvature and Def is urethral defect length in $\mathrm{cm}$. requiring repair. This equation allowed us to assess the ratio of normal urethra that must be mobilized to bridge a gap. As noted, maximal stretched length without penile curvature at artificial erection was a constant factor with a mean value of $75.2 \%$ of maximal constant traction length. Therefore, Un $=0.752$ Tmax - Def. We assessed mean normal urethral variation or extensibility

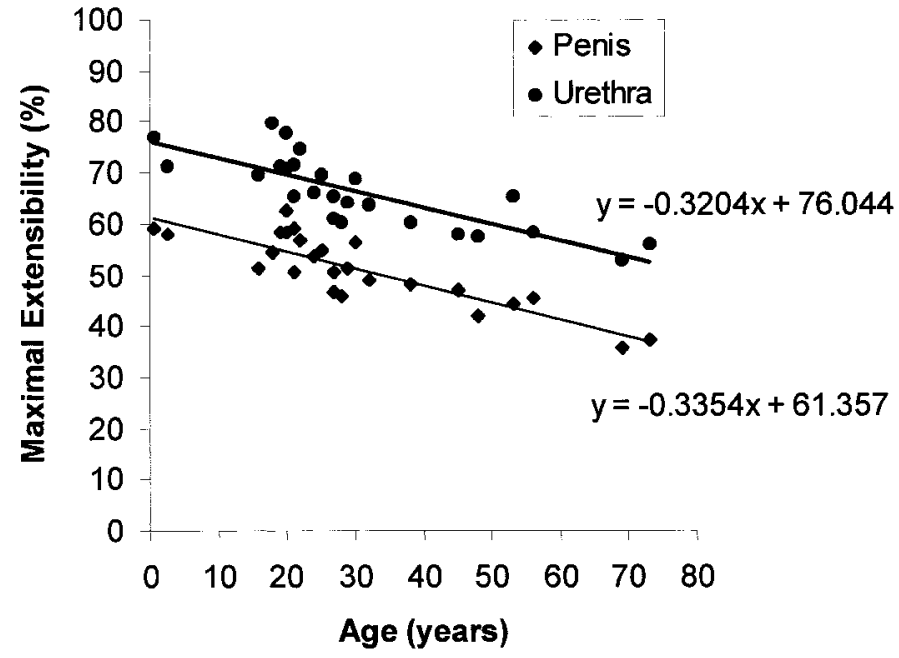

Fig. 1. Correlation of maximal extensibility of human male urethra (thick line) with penis (thin line) by patient age. Urethral extensibility was significantly higher than penile extensibility ( $p$ $<0.001)$ and each decreased with aging $(r=-0.806$ and -0.864 , respectively).

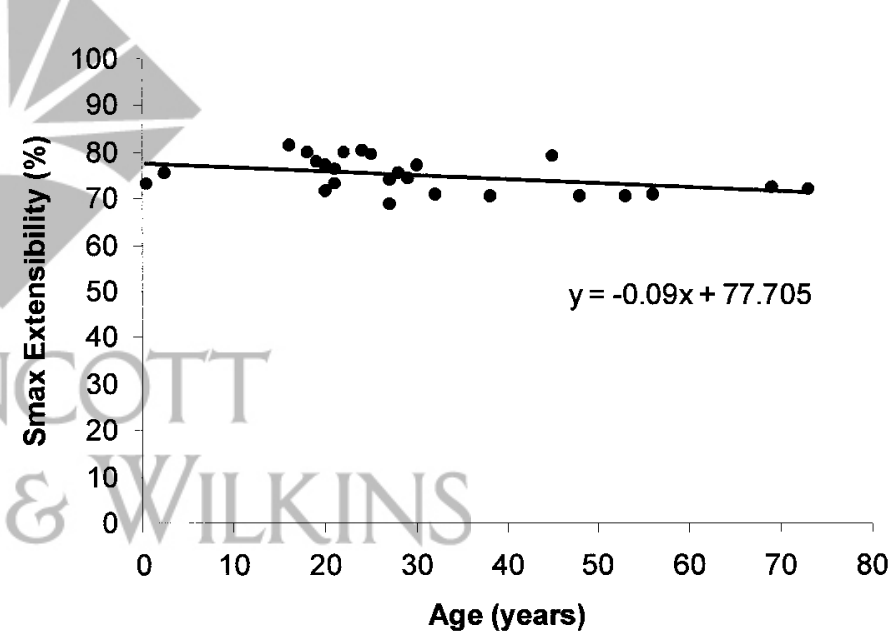

FIG. 2. Correlation of maximal stretched length without inducing curvature at artificial erection (Smax) with patient age. Maximal stretched length slightly decreased with aging $(r=-0.4267, p$ $<0.05)$.

under maximal tension and it was $66.2 \%$ that of the normal urethra. Thus, Un $=(0.752)(1.662 \mathrm{Un})-$ Def $\rightarrow$ Un $=$ 1.2498Un - Def $\rightarrow 1.2498 U n-$ Un $=$ Def $\rightarrow 0.2498 U n=$ Def $\rightarrow \mathrm{Un}=\mathrm{Def} / 0.2498 \rightarrow \mathrm{Un}=4$ Def. Therefore, the ratio is $1: 4$. That is, to bridge a $1 \mathrm{~cm}$. gap $4 \mathrm{~cm}$. of normal urethra mobilization are recommended.

Specifically our study showed that, while maximal stretched length without penile curvature at artificial erection was a constant value, maximal constant traction length varied with age. Thus, using the previous equation and substituting the respective equation of linear estimation for urethral maximal constant traction length $\mathrm{Un}=$

TABLE 1. Length of segments of 25 male urethras with and without maximal constant traction

\begin{tabular}{|c|c|c|c|}
\hline \multirow{2}{*}{ Urethral Segment } & \multicolumn{2}{|c|}{ Mean Length $\pm \mathrm{SD}(\mathrm{cm})}$. & \multirow{2}{*}{ Mean $\%$ Extensibility \pm SD* } \\
\hline & Without Traction & With Traction & \\
\hline Total urethra & $18.6 \pm 5.0$ & $30.7 \pm 8.1$ & $66.2 \pm 7.2$ \\
\hline Penile & $12.7 \pm 3.7$ & $20.5 \pm 5.8$ & $62.6 \pm 7.4$ \\
\hline Bulbar & $3.4 \pm 1.3$ & $5.7 \pm 2.2$ & $65.6 \pm 6.3$ \\
\hline Membranous & $1.4 \pm 0.4$ & $2.2 \pm 0.7$ & $64.8 \pm 6.8$ \\
\hline
\end{tabular}

* Not significant $(\mathrm{p}=0.283)$. 
TABLE 2. Changes in the length of normal urethra necessary to overcome a gap by patient age

\begin{tabular}{cc}
\hline Age & Estimated Linear Regression Ratio \\
\hline 1 & $1: 3.2$ \\
10 & $1: 3.4$ \\
20 & $1: 3.7$ \\
30 & $1: 4.0$ \\
40 & $1: 4.5$ \\
50 & $1: 5.0$ \\
60 & $1: 5.7$ \\
70 & $1: 6.6$ \\
\hline
\end{tabular}

0.75(-0.0032404 age +1.76044$) \mathrm{Un}-$ Def (fig. 1$)$. Thus, we may now assess specific ratios according to patient age (table 2).

\section{DISCUSSION}

There has been great progress in reconstructive surgery of the male urethra in the last decades. Several complex techniques were developed to correct urethral defects. Flaps and organic or synthetic grafts have been used with great variation in the success rate. ${ }^{13}$ However, there is no an ideal substitute for the male urethra and to our knowledge at this time, there is no alternative as good as the urethra. Longterm results of urethroplasty for stricture show that the best success rate is achieved by excising the stricture and forming end-to-end anastomosis of the 2 healthy ends on either side. ${ }^{14}$ Also, surgical techniques of hypospadias repair that use the original urethral tissue rather than flap or graft techniques result in fewer complications. ${ }^{15-17}$

Penile extensibility is limited by the tunica albuginea of the corpora cavernosa. ${ }^{18,19}$ The tunica albuginea of the corpus spongiosum has a thin adventitia and its collagen fibers do not form a complex structure, as do those of the tunica albuginea of the corpora cavernosa. The male urethra has great capacity to extend under traction and we noted that the urethra has greater capacity of extension than the penis. This fact was observed during disassembly surgery for penile lengthening, ${ }^{10}$ in which the main limiting factor is the small capacity of the penile neurovascular bundles to extend and not urethral extensibility.

In the current literature the limits determined for the indication of end-to-end anastomosis in bulbar urethroplasty are exclusively for adults without considering patient age or penis size. These limits are commonly up to $3 \mathrm{~cm} .7,14,20$ Mobilizing the spongy urethra is possible for advancement and does not cause curvature if the limit of $75 \%$ of maximal constant traction length or the general ratio of $1: 4$ is used. Each additional opening in the healthy urethra end (spatulation technique) can be included in defect length. However, as in the penis, ${ }^{18,21}$ urethral extensibility decreases with aging. In older patients the urethra is less extensible than in young patients, which should be considered at surgery. Furthermore, older patients have alterations that interfere negatively with wound healing. In regard to hypospadias we identified only 1 study in which a ratio of 1:3 was empirically recommended. ${ }^{5}$

To enhance the application of our results and decrease adverse effects due to short or extended mobilization of the spongy urethra we recommend using our data (table 2). Structural alterations in the extracellular matrix of the penis with aging should decrease its extensibility. These alterations may be considered a normal event of penile aging. Thus, ideally the limits that we propose should be adjusted to patient age. With respect to the application of the extension principle to the urethra in boys several long-term results confirm its safety. Therefore, the fear of late chordee, ischemia of the anastomosis site or even necrosis is not justified.5,15,16 If the limits proposed in the current study are followed, no mobilization related complications would be expected, such as meatal retraction or dehiscence.

Extrapolating our results in cadavers to live urethras may carry some limitations. However, we studied macroscopically normal, fresh cadaveric urethras, which we consider the most perfect available samples to answer the clinical question of how far the male urethra may be stretched before causing penile curvature. We have previously performed a small pilot study that showed similar urethral extensibility in fresh and thawed specimens. Thus, we believe that freezing does not cause significant changes in the urethral and penile extensibility.

We did not identify any morphological study in the literature that justified previous values. We believe that those values were determined by surgeons with recognized experience in urethral surgery based on their practical clinical and perioperative subjective impressions without rigorous measurement. Also, urethral size was never considered a limiting factor and penile dimensions vary considerably. ${ }^{22}$

Interestingly some groups described successful repair using end-to-end anastomosis after the failure of a previous operative attempt.7,20 In this situation it is more logical to consider that maximal stretched length without penile curvature at artificial erection was not explored at previous surgery rather than that a proliferation of extracellular matrix was stimulated by tension. However, additional factors can limit the use of the extension principle in urethral surgery. Extensive mobilization of the male urethra may injure the urethral blood supply and spongiosclerosis or vascular erectile dysfunction may develop. ${ }^{23,24}$ Also, according to the law of Laplace over stretching the male urethra decreases its lumen and may cause high bladder pressure during voiding. ${ }^{25}$

Despite differences in structure the whole urethra and each urethral segment have similar extensibility. The impression that the bulbar urethra has more longitudinal extensibility than other urethral segments is not true. ${ }^{9} \mathrm{We}$ noted that longitudinal extensibility of the bulbar urethra is the same as that of the other segments. The distribution of corpus spongiosum in the bulbous urethra causes high extensibility in the axial plane, permitting great dilatation of its lumen. What is relevant to surgeons is that bulbar mobilization may correct the natural angle of curvature of the bulbar urethra to gain additional length. Furthermore, other alternative procedures to achieve more correction have been described, such as splitting the corpora cavernosa and excising the suspensory ligament, which in addition to extension properties, may overcome large urethral defects without grafts or flaps. ${ }^{14}$

\section{CONCLUSIONS}

Knowledge of the safe anatomical limit of urethral extensibility applied to reconstructive surgery may avoid complications and the necessity of more complex techniques. This limit should be approximately $75 \%$ of maximal constant traction length or a general gap-to-normal urethra ratio of 1:4. However, age related variations of urethral extensibility should be considered. Thus, the ratio in younger patients is lower than in older patients.

Dr. Carlos A. B. Klojda provided most anatomical specimens.

\section{REFERENCES}

1. Hsu, G. L., Brock, G., von Heyden, B. et al: The distribution of elastic fibrous elements within the human penis. Br J Urol, 73: 566, 1994

2. Beck, C.: Hypospadias and its treatment. Surg Gynecol Obstet, 24: 511,1917

3. Badenoch, A. W.: A pull-through operation for impassable traumatic stricture of the urethra. Br J Urol, 22: 404, 1950

4. Koff, S. A.: Mobilization of the urethra in the surgical treatment 
of hypospadias. J Urol, 125: 394, 1981

5. Hamdy, H., Awadhi, M. A. and Rasromani, K. H.: Urethral mobilization and meatal advancement: a surgical principle in hypospadias repair. Pediatr Surg Int, 15: 240, 1999

6. Mitchell, M. and Bagli, D. J.: Complete penile disassembly for epispadia repair: the Mitchell technique. J Urol, 155: 300, 1996

7. Martinez-Pineiro, J. A., Carcamo, P., Garcia-Matres, M. J. et al: Excision and anastomotic repair for urethral stricture disease: experience with 150 cases. Eur Urol, 32: 433, 1997

8. Perovic, S. V., Vukadinovic, V., Djordjevic, M. L. J. et al: The penile disassembly technique in hypospadias repair. Br J Urol, 81: 479, 1998

9. Turner-Warwick, R., Parkhouse, H. and Chapple, C. R.: Bulbar elongation anastomotic meatoplasty (BEAM) for subterminal and hypospadiac urethroplasty. J Urol, 158: 1160, 1997

10. Perovic, S. V. and Djordjevic, M. L. J.: Penile lengthening. BJU Int, 86: 1028,2000

11. Gittes, R. F. and McLaughlin, A. P.: Injection technique to induce penile erection. Urology, 4: 473, 1974

12. Kelami, A.: Classification of congenital and acquired penile deviation. Urol Int, 38: 229, 1983

13. Chapple, C. and Turner-Warwick, R.: Substitution urethroplasty and the pedicled island penile skin procedure. In: Traumatic and Reconstructive Urology. Edited by J. W. McAninch. Philadelphia: W. B. Saunders, chapt. 48, pp. 571-594, 1996

14. Andrich, D. E. and Mundy, A. R.: Urethral strictures and their surgical treatment. BJU Int, 86: 571, 2000

15. Caione, P., Capozza, N., Lais, A. et al: Long-term results of distal urethral advancement glanuloplasty for distal hypospadias.
J Urol, 158: 1168, 1997

16. De Sy, W. A. and Hoebeke, P.: Urethral advancement for distal hypospadias: 14 years' experience. Eur Urol, 26: 90, 1994

17. Snodgrass, W., Koyle, M., Manzoni, G. et al: Tubularized incised plate hypospadias repair for proximal hypospadias. J Urol, 159: 2129, 1998

18. De Goes, P. M., Wespes, E. and Schulman, C.: Penile extensibility: to what is it related? J Urol, 148: 1432, 1992

19. Bitsch, M., Kromann-Andersen, B., Schou, J. et al: The elasticity and the tensile strength of tunica albuginea of the corpora cavernosa. J Urol, 143: 642, 1990

20. Morey, A. F., Duckett, C. P. and McAninch, J. W.: Failed anterior urethroplasty: guidelines for reconstruction. J Urol, 158: 1383, 1997

21. Bondil, P., Costa, P., Daures, J. P. et al: Clinical study of the longitudinal deformation of the flaccid penis and of its variations with aging. Eur Urol, 21: 284, 1992

22. Schneider, T., Sperling, H., Lummen, G. et al: Does penile size in younger men cause problems in condom use? A prospective measurement of penile dimensions in 111 young and 32 older men. Urology, 57: 314, 2001

23. Droupy, S., Giuliano, F., Jardin, A. et al: Cavernospongious shunts: anatomical study of intrapenile vascular pathways. Eur Urol, 36: 123, 1999

24. Coursey, J. W., Morey, A. F., McAninch, J. A. et al: Sexual outcomes of anterior urethroplasty. J Urol, suppl., 163: 70A, abstract 306, 2000

25. Plevnik, S. and Janez, J.: Pressure-distension relation in male obstructed urethra. Urology, 17: 393, 1981

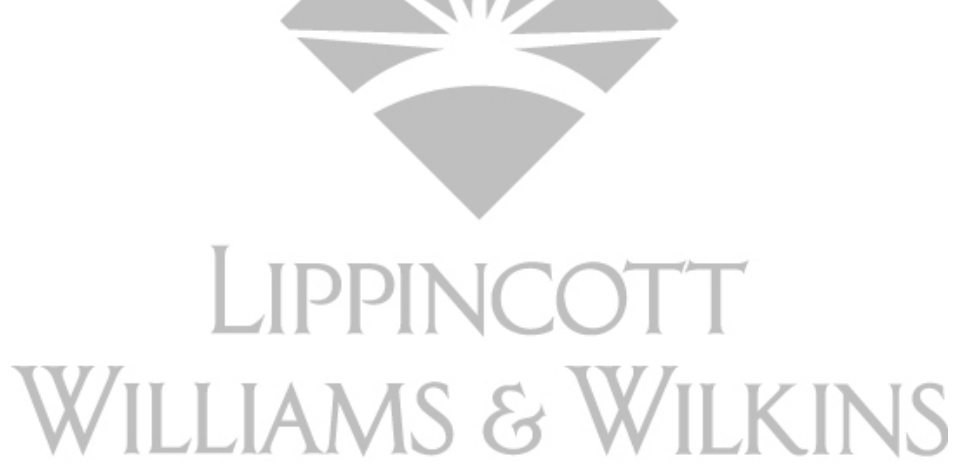

